\title{
Editorial commentary on the Indian Journal of Gastroenterology—January-February 2022
}

\author{
Jimmy K Limdi ${ }^{1,2,3}$ (D) \\ Published online: 21 February 2022 \\ (C) Indian Society of Gastroenterology 2022
}

\section{Impact of delay in diagnosis in patients with celiac disease: A study of $\mathbf{5 7 0}$ patients at a tertiary care center}

The clinical presentation of celiac disease (CD) is highly variable, ranging from gastrointestinal symptoms from malabsorption, extraintestinal manifestations to being asymptomatic. Consequently, a significant proportion of patients may remain undiagnosed, be misdiagnosed, or experience diagnostic delays [1]. The implications of delayed diagnosis are less well studied. Dhar et al. from the Postgraduate Institute of Medical Education and Research, Chandigarh, India, report a retrospective analysis of 570 individuals with CD [2]. Patients were divided into an early-diagnosis ( $\leq 3$ years; 289 patients) or a late-diagnosis ( $>3$ years; 281 patients) group. Patients in the late-diagnosis group had lower rates of diarrhea as the presenting symptom but higher rates of anemia, pubertal delay, and menstrual irregularities as well as laboratory abnormalities with lower hemoglobin, ferritin, and vitamin D levels and higher anti-tissue transglutaminase antibody titers with higher grades of villous atrophy at presentation. Among unmet needs for $\mathrm{CD}$, physician awareness of diagnostic criteria, improved access to specialist assessment, and the impact of education about gluten-free eating as well as access and

Jimmy K Limdi

Jimmy.Limdi@manchester.ac.uk; Jimmy.Limdi@nhs.net

1 Section of Inflammatory Bowel Diseases, Division of Gastroenterology, The Northern Care Alliance NHS Foundation Trust, Manchester, UK

2 Manchester Academic Health Sciences, University of Manchester, Manchester, UK

3 Manchester Metropolitan University, Manchester, UK availability of gluten-free foods to support adherence need further study.

\section{The role of gut microbiota in clinical complications, disease severity, and treatment response in severe alcoholic hepatitis}

In addition to the direct effects of alcohol on the liver, indirect effects have also been explored with recent focus on the relationship between alcohol, the liver, and gut microbiota. Dynamic changes of intestinal microorganisms play a pivotal role in the development of liver diseases through the integration of signals generated by dietary, genetic, and environmental factors [3].

Philips and colleagues from Ernakulam Medical Center, Kochi, India, aimed to characterize bacterial communities associated with clinical events (CE), to identify significant bacteria linked to $\mathrm{CE}$, and to define bacterial relationships associated with specific $\mathrm{CE}$ and outcomes at baseline and after treatment in severe alcoholic hepatitis (SAH) using 16-s rRNA sequencing on stool samples collected at admission and the last follow-up within 90 days in 38 patients with SAH [4].

They noted specific associations between bacterial families and adverse outcomes: e.g. Lachnobacterium and Catenibacterium with hepatic encephalopathy, Pediococcus with death after steroid treatment, and a change from Enterococcus to Barnesiella after granulocyte colony-stimulating factor (G-CSF) therapy. Future research aimed at further characterization and the designing of a broad-spectrum bacteriophage therapy to prevent and treat diseases related to microbiota imbalance in 
patients with alcohol-related liver disease is needed to address the limited therapeutic options that existed for this disease.

\section{Effect of long-term aggressive nutrition therapy on survival in patients with alcohol-related cirrhosis: A randomized controlled trial}

An adequate calorie and protein intake is difficult to achieve in malnourished patients with advanced liver disease [5]. In patients with malnutrition and alcoholic liver cirrhosis (ALC), who are unable to achieve adequate dietary intake with an oral diet, short-term enteral or parenteral nutrition is often attempted to overcome the phase of underfeeding. The effect of higher calorie and protein intake on survival is less well studied. Kalal and colleagues from the Institute of Liver and Biliary Sciences, New Delhi, India, report a randomized controlled trial of malnourished patients with ALC, assessed by Royal Free Hospital-Subjective Global Assessment (RFH-SGA) [6]. Patients were randomized to a control group (CG, $n=50$; 35$40 \mathrm{kcal}$ and $1.2 \mathrm{~g}$ protein $/ \mathrm{kg} /$ day diet alone) or an intervention group (IG; $n=54,40-45 \mathrm{kcal}$ and $1.5 \mathrm{~g}$ protein $/ \mathrm{kg} /$ day diet plus polymeric formula) for 3 months. The RFH-SGA improved in $33.3 \%$ in IG vs. $14.2 \%$ in CG at 3 months, with better resolution of ascites in the IG. Although median 12-month survival was comparable between groups, energy intake $>25 \mathrm{kcal}$ and protein $>0.8 \mathrm{~g} / \mathrm{kg} /$ day significantly improved 12 -month survival. Screening and management of nutrition in ALC remains a challenging but integral aspect of care of these patients.

\section{Screening for non-alcoholic fatty liver disease among obese and overweight children: Prevalence and predictors}

Non-alcoholic fatty liver disease (NAFLD) is one of the most common hepatic diseases in children with particular risk factors including obesity, sedentary lifestyle, and/or a predisposing genetic background [7]. The worldwide prevalence of NAFLD in children is worryingly increasing and concerning because this disease is closely associated with the development of both cirrhosis and cardiometabolic syndrome in adulthood. The etiopathogenesis of primary NAFLD in children is unknown. Srinivasan and colleagues from the Indira Gandhi Medical College and Research Institute, Puducherry, India, report a cross-sectional study of 154 overweight and obese children, divided into 2 groups based on the presence or absence of NAFLD [8]. NAFLD was noted in $51.3 \%$ of these children and was associated with high low-density lipoprotein (LDL) cholesterol levels. The authors highlight the need to screen high-risk children for NAFLD. Indeed, understanding the pathogenetic mechanisms of NAFLD will form the basis for the characterization of early predictors of the disease and the development of non-invasive diagnostic tools and the designing of novel specific treatments and management strategies.

\section{Normative values of skeletal muscle indices for nutritional assessment and implications on the definition of sarcopenia in the Indian adult population}

Sarcopenia is a clinical condition in which skeletal muscle mass and strength gradually decline and have the potential for adverse outcomes including poor quality of life, disability, and death. It has a prevalence range of $5 \%$ to $13 \%$ in people 60-70 years of age and $11 \%$ to $50 \%$ in people $>80$ years [9]. Screening and diagnosis of sarcopenia are important to prevent adverse health outcomes. Computerized tomography (CT) and magnetic resonance imaging (MRI) are the gold standards for noninvasively assessing muscle quantity/mass and identifying adipose tissue. Choudhary and colleagues from Dr. B. L. Kapur Memorial Hospital, New Delhi, India, report a cross-sectional quantitative analysis of various skeletal muscle indices including psoas and erector spinae muscles, aiming to define sarcopenia [10]. Further validation and determination of baseline values may serve as a reference point for assessment and future studies of sarcopenia with important clinical implications.

\section{Deficiency of folate and vitamin $B_{12}$ increases oxidative stress in chronic pancreatitis patients}

Activation of pancreatic stellate cells (PSCs) is a key antecedent of fibrosis and hallmark of chronic pancreatitis (CP). Repetitive oxidative stress transforms the fat-storing PSCs into myofibroblast-like cells, capable of producing chemokines, and adhesion molecules in response to inflammatory infiltration. Levels of several antioxidants (e.g. selenium, ascorbic acid $\beta$-carotene, $\alpha$-tocopherol, and methionine) are deficient in many forms of oxidative stress [11].

Banavara and colleagues from the Amrita Institute of Medical Sciences, Kochi, India, measured erythrocyte folate and plasma vitamin B and compared these with blood antioxidants - erythrocyte glutathione (GSH), glutathione peroxidase (GPx), superoxide dismutase (SOD), plasma vitamin $\mathrm{C}$, and markers of lipid peroxidation, thiobarbituric acid reactive substance (TBARS), in CP patients [12].

Among 175 CP patients (91 tropical, 84 alcoholic) and 113 healthy controls, erythrocyte folate plasma vitamin $\mathrm{B}_{12}$ and blood antioxidant levels were significantly lower in $\mathrm{CP}$ patients than in controls and in diabetic vs. non-diabetic $\mathrm{CP}$ 
patients. $\mathrm{CP}$ patients with low folate and vitamin $\mathrm{B}_{12}$ had low GSH and GPx levels as compared to patients with normal folate and vitamin $B_{12}$ levels. Low vitamin $B_{12}$ level was associated with 3.24-fold increased risk of pancreatic insufficiency. The role of $\mathrm{B}_{12}$ and folate supplementation and its impact on improvement in antioxidant levels in supplemented patients, improvement in organ dysfunction, clinical outcomes, or reduced length of stay need further study.

\section{Health and economic burden due to alcohol-associated liver diseases in the Union Territory of Delhi: A Markov probabilistic model approach}

The multidimensional and negative impact of alcohol misuse on socioeconomic and health outcomes is less well appreciated. Ramalingam and colleagues from the Institute of Liver and Biliary Sciences, New Delhi, India, assessed the health and economic burden due to alcohol-associated liver disease (ALD) for Delhi, between March 2017 and February 2018, using a Markov probabilistic model [13]. They estimated disability adjusted life years (DALYs), years of life lost (YLL), and total deaths due to ALD [13]. Their findings are unsettling: there were 8367 estimated deaths due to ALD. DALYs due to ALD were estimated at 0.247 million life years, with 0.178 million YLL and 0.069 million life years lost due to disability. The total cost of treating ALD was estimated to be 92.94 billion Indian rupees, if patients sought care based on current preferences, and 55.52 billion Indian rupees assuming all diseased individuals were cared for in public health systems. Their data are a wake-up call for socioeconomic strategies to mitigate against the huge burden to individuals and the nation.

\section{Small-intestinal bacterial overgrowth in patients with inflammatory bowel disease: A case-control study}

Despite its greater prevalence, small-bowel bacterial overgrowth (SIBO) in patients with inflammatory bowel disease (IBD) is often overlooked. SIBO has a positive correlation with worsening of abdominal symptoms such as bloating, flatus, satiety, and loose stools in IBD [14]. Ghoshal and colleagues, from the Sanjay Gandhi Postgraduate Institute of Medical Sciences, Lucknow, India, report a retrospective study of 86 IBD patients (45 with ulcerative colitis [UC]) undergoing glucose hydrogen breath test (GHBT) to evaluate the frequency and risk factors of SIBO compared to 66 healthy controls [15]. A higher prevalence of SIBO was noted in Crohn's disease than in UC (34.1 vs. $2 / 45$ [4.4\%]; $p=$
0.001). The prevalence of SIBO in UC was comparable to that in controls. Female gender and surgery for IBD were independent factors associated with SIBO among IBD patients on multivariate analysis. Clinicians must maintain an appropriate index of suspicion to diagnose and manage SIBO, which can be associated with significant morbidity in IBD.

\section{The challenges of implementing a low-fermentable oligo-, di-, and monosaccharide and polyol diet in India: An analysis of available data}

The elimination of dietary fermentable oligosaccharides, disaccharides, monosaccharides, and polyols (FODMAPs) has rapidly gained popularity as a treatment for patients with irritable bowel syndrome (IBS) [16]. FODMAPs lead to increased gastrointestinal (GI) water secretion and increased fermentation in the colon, thus producing short-chain fatty acids and gasses, which can lead to luminal distension and the triggering of meal-related symptoms in patients with IBS. The complexity of the low-FODMAP diet, combined with the potential for nutritional deficiencies, taken together with the time and resources required to provide proper counseling, poses significant challenges. Mustafa and Ghoshal, from the Sanjay Gandhi Postgraduate Institute of Medical Sciences, Lucknow, India, report a thoughtprovoking study on the challenges posed by the implementation of a FODMAP-restricted diet in India [17].

Using data on dietary patterns of Indian states and union territories, from the vital statistics database maintained by the Indian government (Census and National Family Health Survey), PubMed, and Google Scholar, the prevalence of vegetarianism in India was computed. There is a high prevalence of vegetarianism with regional variation in the frequency of lactose malabsorption. Although southern, eastern, and northern India have rice as the staple cereal, wheat is consumed in higher proportions in other parts of India. The authors discuss geographical differences and challenges therein, with implementation of a low-FODMAP diet across India.

\section{Declarations}

Conflict of interest JKL declares that he has no conflict of interest.

Disclaimer The author is solely responsible for the data and the contents of the paper. In no way is the honorary editor in chief, editorial board members, the Indian Society of Gastroenterology or the printer/publishers responsible for the results/findings and content of this article. 


\section{References}

1. Cichewicz AB, Mearns ES, Taylor A, et al. Diagnosis and treatment patterns in celiac disease. Dig Dis Sci. 2019;64:2095-106.

2. Dhar J, Samanta J, Sharma M, et al. Impact of delay in diagnosis in patients with celiac disease: A study of 570 patients at a tertiary care center. Indian J Gastroenterol. 2022;41. https://doi.org/10.1007/ s12664-021-01214-3.

3. Tilg H, Cani PD, Mayer EA. Gut microbiome and liver diseases. Gut. 2016;65:2035-44.

4. Philips CA, Augustine P, Ganesan K, et al. The role of gut microbiota in clinical complications, disease severity, and treatment response in severe alcoholic hepatitis. Indian J Gastroenterol. 2022;41. https://doi.org/10.1007/s12664-021-01157-9.

5. European Association for the Study of the Liver. EASL clinical practice guidelines on nutrition in chronic liver disease. J Hepatol. 2019;70:172-93.

6. Kalal C, Benjamin J, Shasthry V, et al. Effect of long-term aggressive nutrition therapy on survival in patients with alcohol related cirrhosis: A randomized controlled trial. Indian J Gastroenterol. 2022;41. https://doi.org/10.1007/s12664-021-01187-3.

7. Mann JP, Valenti L, Scorletti E, et al. Nonalcoholic fatty liver disease in children. Semin Liver Dis. 2018;38:1-13.

8. Thiagarajan S, Shrinuvasan S, Babu TA. Screening for nonalcoholic fatty liver disease among obese and overweight children: Prevalence and predictors. Indian J Gastroenterol. 2022;41. https:// doi.org/10.1007/s12664-021-01198-0.

9. Supriya R, Singh KP, Gao Y, et al. A multifactorial approach for sarcopenia assessment: a literature review. Biology (Basel). 2021;10:1354.

10. Choudhary A, Wadhawan M, Dhawan S, et al. Normative values of skeletal muscle indices for nutritional assessment and implications on definition of sarcopenia in Indian adult population. Indian $\mathrm{J}$ Gastroenterol. 2022;41. https://doi.org/10.1007/s12664-02101207-2.

11. Swentek L, Chung D, Ichii H. Antioxidant therapy in pancreatitis. Antioxidants (Basel). 2021;10:657.

12. Girish BN, Rajesh G, Vaidyanathan K. Deficiency of folate and vitamin $\mathrm{B}_{12}$ increases oxidative stress in chronic pancreatitis patients. Indian J Gastroenterol. 2022;41. https://doi.org/10.1007/ s12664-021-01210-7.

13. Ramalingam A, Pasupuleti SSR, Nagappa B, Sarin SK. Health and economic burden due to alcohol associated liver diseases in the Union Territory of Delhi: A Markov probabilistic model approach. Indian J Gastroenterol. 2022;41. https://doi.org/10.1007/s12664021-01221-4.

14. Colombel JF, Shin A, Gibson PR. AGA Clinical practice update on functional gastrointestinal symptoms in patients with inflammatory bowel disease: expert review. Clin Gastroenterol Hepatol. 2019;17: 380-90.e1.

15. Ghoshal UC, Yadav A, Fatima B, et al. Small intestinal bacterial overgrowth in patients with inflammatory bowel disease: A casecontrol study. Indian J Gastroenterol. 2022;41. https://doi.org/10. 1007/s12664-021-01211-6.

16. Lacy BE, Pimentel M, Brenner DM, et al. ACG clinical guideline: management of irritable bowel syndrome. Am J Gastroenterol. 2021;116:17-44.

17. Mustafa U, Ghoshal UC. The challenges of implementing low fermentable oligo-, di-, mono-saccharides and polyol diet in India: An analysis of available data; 2022;41. https://doi.org/10.1007/s12664022-01250-7.

Publisher's note Springer Nature remains neutral with regard to jurisdictional claims in published maps and institutional affiliations. 